\title{
Approach to Assessment of Risk Factors in Mild Hypertension
}

\author{
K. L. STUART, PATRICIA DESAI, ADELLA LALSINGH
}

British Medical fournal, 1974, 2, 195-198

\section{Summary}

Criteria are urgently needed for the early detection of subjects with only mildly raised blood pressure who may be at high risk of developing the complications of hypertension. As a step towards the establishment of such criteria we have examined the association of certain possible "risk" factorsnamely, $x$-ray evidence of cardiac enlargement, high serum cholesterol levels, effort pain, E.C.G. abnormalities, and high systolic blood pressure-with fatal or morbid endpoints in a five-year follow-up study of subjects whose diastolic pressure had been found initially to be between 95 and $114 \mathrm{~mm} \mathrm{Hg}$. The index group consisted of 22 patients in whom these endpoints occurred. They comprised death from cardiovascular disease, clinical or E.C.G. deterioration, and either an increase in diastolic pressure of at least $10 \mathrm{~mm} \mathrm{Hg}$ or a diastolic pressure of $115 \mathrm{~mm} \mathrm{Hg}$ or both. The control group consisted of 22 subjects chosen at random from other respondents with the same range of diastolic pressures and the same age and sex distribution.

"Any two or more" of the possible risk factors examined were found to occur significantly more often in the index group than in the controls, suggesting a possible approach to the early detection of high-risk subjects. The value of longterm studies along these lines and the urgent need for them are emphasized.

\section{Introduction}

Patients with severe or moderate hypertension are known to benefit from treatment. Whether patients with mild hypertension would derive the same benefits, however, is not known, though Smirk (1957) and the Veterans Administration Cooperative Study Group on Anti-hvpertensive Agents (1967, 1970) have suggested that they might. Identification of such subjects and their routine treatment, however, would impose serious economic burdens on most communities and put large numbers of people to personal inconvenience.

All communities contain people with diastolic blood pressures of over $115 \mathrm{~mm} \mathrm{Hg}$ but the number with diastolic pressures of $95-114 \mathrm{~mm} \mathrm{Hg}$ must be several times greater. There is certainly a higher risk of death from vascular disease, especially mvocardial infarction and stroke, in subjects with the higher levels, but the Society of Actuaries (1959) has shown that these complications occur mainly in persons with mild hypertension. This finding accords also with the observations of Evans and Rose (1971), that the main burden of illness associated with high blood pressure arises not from the relatively few severe cases but from the very large number of people with pressures considered to be only mildly raised. The recognition and attempted treatment, however, of all subjects with mild hypentension in large communities would

University of the West Indies, Kingston, Jamaica K. L. STUART, M.D., F.R.C.P., Professor of Medicine PATRICIA DESAI, B.A., Statistician, M.R.C. Epidemiology Unit ADELLA LALSINGH, Medical Student pose problems of both practicability and ethical justification. Thus two major questions would have to be answered before routine treatment of this group of people could be instituted. The first relates to the recognition of subjects with mild hypertension who may be at greatest risk, and the second is whether these high-risk subjects once identified could be shown to benefit from lowering of the blood pressure. Logically the first question would have to be settled before embarking on the large and long-term studies that would be required to answer the second.

In this preliminary study we analysed some of the factors that may be of value in identifying high-risk subjects. We followed for five years a number of subjects who were found initially to have mild hypertension. Some remained symptomfree and showed no cardiovascular deterioration, whereas others showed appreciable increases in blood pressure or developed one or more of the recognized complications of hypertension. The results of a number of observations at the time of the initial blood pressure survey were then reviewed to see which of these or which combination of these would have been of most value in predicting the outcome.

\section{Subjects and Methods}

A longitudinal study of subjects with mild hypertension (diastolic blood pressure $95-114 \mathrm{~mm} \mathrm{Hg}$ ) was begun in a typically rural population sample in Jamaica in 1962. The outcome in these subjects in 1967 was then related to the presence of certain possible "risk" factors. These were evidence of an enlarged heart, high serum cholesterol levels, effort pain, E.C.G. abnormalities, and high systolic blood pressure. The subjects were chosen at random from a total population of about 7,500 living in a geographically defined area at Lawrence Tavern, about 16 miles $(26 \mathrm{~km})$ nonth of Kingston. The population was typically rural and was enumerated by private census during March to July 1962. The results of an epidemiological study of the prevalence of heart disease in this population (Fodor et al., 1964) and of longitudinal follow-up studies (Miall et al., 1972) of these and a further sample of respondents have already been published.

Altogether 600 subjects in the age ranges 35-44, 45-54, and 55-64 years were chosen for study initially, and the response rate at that time was $91.3 \%$ (548 subjects). At the five-year follow-up 434 subjects were re-examined $(79.2 \%$ of the 548 ; $72.3 \%$ of the original 600 ).

Blood pressure was recorded by sphygmomanometry in the right arm at a rural health centre after the subjects had been sitting for 10 minutes. Serum cholesterol was measured by the method of Zlatkis, Zak, and Boyle using MacIntyre and Ralston's (1954) modification. The questionnaire on effort pain was designed by Rose (1962). The chest $x$-ray pictures were taken during 1964-5. Characteristics sought on the E.C.G.s were evidence of left ventricular hypertrophy by voltage criteria (Sokolow and Lyon, 1949) or ST-T abnormalities or both. Chest $x$-ray films and E.C.G.s were assessed without knowledge of the other symptoms, laboratory findings, or subsequent medical history.

This analysis is concerned solely with respondents whose diastolic pressure (phase IV) in 1962 was in the range 95-114 
$\mathrm{mm} \mathrm{Hg}$. Altogether 101 persons were in this group. The analysis compares "index" and "control" groups.

The index group consisted of 22 subjects in the following categories: (a) death from cardiovascular causes between the 1962 and 1967 surveys ( 3 cases), (b) illness due to cardiovascular disease (10 cases), (c) a rise in diastolic pressure of at least $10 \mathrm{~mm} \mathrm{Hg}$ between 1962 and 1967 and/or a rise to a level of $115 \mathrm{~mm} \mathrm{Hg}$ or more by 1967 (12 cases). Three persons were in both $(b)$ and $(c)$ categories. The age and sex distribution of the index cases was as follows. 35-44 years 1 man; 45-54 years 2 men, 7 women; and 55-64 years 6 men, 6 women. The three cardiovascular deaths were due to cerebral haemorrhage (2) and hypertensive cardiac failure (1). The 10 morbid events of cardiovascular origin were E.C.G. deterioration (5), heart failure (3), and heart disease and effort pain (2). The E.C.G. deterioration consisted of changes from minor ST-T abnormalities to marked $T$-wave inversion with $S-T$ segment depression in four cases and the development of frequent ventricular ectopic beats in the fifth.

The control group consisted of 22 subjects chosen at random from other respondents with the same range of diastolic pressures and the same age and sex distribution.

The means and standard deviations of the initial diastolic pressures were $103.9 \pm 5.5 \mathrm{~mm} \mathrm{Hg}$ in the index group and $103.2 \pm 5.3 \mathrm{~mm} \mathrm{Hg}$ in the control group.

\section{Results}

The index and control groups were tested separately for differences in the prevalence of each risk factor (table I). There was almost no difference between the groups with regard to numbers with high cholesterol values. This was confirmed by calculation of the mean values for each group, which were not significantly different. Effort pain, a high cardiothoracic ratio, and E.C.G. abnormalities all occurred more often in the index group than in the control group, but no statistically significant association was reached when each of these factors was assessed separately. The differences between the index and control groups with regard to high systolic pressure were significant (one-tailed $\chi^{2}$ test : $P<0.05$ ). Comparison of the mean pressures, however, showed no significant difference between the groups, though the mean systolic pressures were $10 \mathrm{~mm} \mathrm{Hg}$ higher in the index group $(169 \pm 29.6 \mathrm{~mm} \mathrm{Hg})$ than in the controls (159 \pm $18.2 \mathrm{~mm} \mathrm{Hg}$ ).

TABLE I-Presence of Risk Factors in Index and Control Groups. Figures are Numbers of Subjects

\begin{tabular}{|c|c|c|c|c|c|c|}
\hline \multirow[b]{2}{*}{ Risk Factors } & \multicolumn{3}{|c|}{ Index Group $(n=22)$} & \multicolumn{3}{|c|}{ Control Group $(n=22)$} \\
\hline & Present & Absent & $\underset{\text { Known }}{\text { Not }}$ & Present & Absent & $\begin{array}{c}\text { Not } \\
\text { Known }\end{array}$ \\
\hline 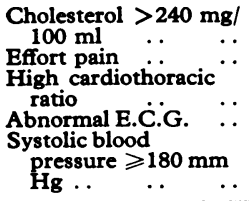 & $\begin{array}{r}7 \\
7 \\
6 \\
11 \\
\\
10\end{array}$ & $\begin{array}{r}11 \\
15 \\
8 \\
11 \\
\\
12\end{array}$ & 4 & $\begin{array}{l}8 \\
2 \\
2 \\
5\end{array}$ & $\begin{array}{r}11 \\
20 \\
6 \\
17\end{array}$ & 3 \\
\hline Any 2 or more & 14 & 5 & 3 & 5 & 10 & 7 \\
\hline
\end{tabular}

Analysis was also made of associations between risk factors and differences between the index and control groups with regard to prevalence of each pair of factors. All possible combinations of pairs of factors were considered, making 10 pairs in all. There was almost no difference between the index and control groups when cholesterol was one factor of the pair.
With all the other pairs the prevalence was higher in the index group but not significantly so.

Differences between the groups with regard to the total number of risk factors showed that there was a statistically significant difference between the two groups when examined for the presence of two or more risk factors (table I).

When the two groups were combined and analyses were performed to find whether one factor tended to occur in conjunction with another it was found that the only significant association was between abnormal E.C.G.s and systolic pressures of $180 \mathrm{~mm} \mathrm{Hg}$ or more (table II).

TABLE II-Association between E.C.G. and Systolic Blood Pressure in 44 Respondents

\begin{tabular}{|c|c|c|c|c|c|}
\hline \multirow{2}{*}{ E.C.G. } & & & \multicolumn{2}{|c|}{ Systolic Pressure } & \multirow[b]{2}{*}{ Total } \\
\hline & & & $\geqslant 180 \mathrm{~mm} \mathrm{Hg}$ & $<180 \mathrm{~mm} \mathrm{Hg}$ & \\
\hline $\begin{array}{l}\text { Abnormal } \\
\text { Normal }\end{array}$ & $\therefore$ & $\begin{array}{l}\cdots \\
\cdots\end{array}$ & $\begin{array}{l}9 \\
4\end{array}$ & $\begin{array}{r}7 \\
24\end{array}$ & $\begin{array}{l}16 \\
28\end{array}$ \\
\hline \multicolumn{3}{|c|}{ Total } & 13 & 31 & 44 \\
\hline
\end{tabular}

\section{SENSITIVITY AND SPECIFICITY}

The above results indicate that high systolic pressure or the presence of at least two risk factors may be the best predictors of five-year morbidity or mortality. Their relative merits may be assessed by considering their sensitivity and specificity (table III)-sensitivity $=\%$ of index cases with factor; specificity $=\%$ of control cases without factor.

TABLE III-Sensitivity and Specificity of Predictors: Observed Values with 95\% Confidence Limits

\begin{tabular}{ll|c|c}
\hline Predictor & Sensitivity & Specificity \\
\hline $\begin{array}{ll}\text { High systolic pressure } \\
\text { At least 2 risk factors }\end{array}$ & $\cdots$ & $\begin{array}{l}45 \%(25 \% \text { to } 67 \%) \\
74 \%(48 \% \text { to } 95) \%\end{array}$ & $\begin{array}{l}86 \%(64 \% \text { to } 97 \%) \\
67 \%(38 \% \text { to } 87 \%)\end{array}$ \\
\hline
\end{tabular}

The presence of at least two risk factors showed a sensitivity and specificity of $74 \%$ and $67 \%$ respectively. This predictor, therefore would select $74 \%$ of the index cases but would also include $33 \%$ of the controls (that is $100 \%-67 \%$ ). A high systolic pressure was present in only $45 \%$ of the index cases but showed a high specificity, being absent in $86 \%$ of the controls. These figures and their $95 \%$ confidence limits, the probable ranges within which the true population values lie, are shown in table III.

\section{TREATMENT}

Most of these patients were free of symptoms, and in most cases the blood pressure, though raised, was not at the level for which treatment is normally recommended in our clinics. Only a small number of subjects, therefore, with symptoms not necessarily related to hypertension or at the higher levels of the blood pressure range selected for study were invited to attend the clinic in the area for antihypertensive therapy, and only a few of these actually attended.

In the index group only one patient received regular treatment for the full five-year period of follow-up. One received regular treatment for the first three years and then defaulted. Treatment was intermittent in two further cases. In the control group three subjects received only intermittent treatment. One received regular treatment for the full five-year period.

In none of the patients receiving either intermittent or regular treatment, whether in the index or control group, was satisfactory blood pressure control achieved for any appreciable 
period. It is assumed, therefore, that treatment did not significantly influence the outcome in the subjects followed up in this study.

\section{Discussion}

Of the predictors examined a raised systolic pressure was the only one found separately to be significantly more prevalent in the index group. The others, apart from a high cholesterol level, were all commoner in the index but the numbers were too small for statistically significant differences to be achieved when each predictor was tested individually. Significant differences, however, were shown when two or more of the factors were combined. The results were influenced by a higher number of risk factors in older people and a higher number among women than among men.

Because of the level of systolic pressure is not commonly used as an indication for treatment, and because most treatment regimens have their greatest effects on the diastolic pressure, the latter has become the main indication for treatment and the main measure of its effectiveness. The significance of the levels of systolic pressure is therefore of ten overlooked, though it is well recognized that high levels are associated independently with an adverse prognosis (Evans and Rose, 1971).

Failure to show an association between higher cholesterol levels and morbid or fatal endpoints may be due to our small numbers and the relatively short follow-up period. Oglesby Paul (1971) showed in a 10-year follow-up study of 6,640 men aged 30-59 years that when first seen those with mild hypertension whose serum cholesterol concentrations were 255 $\mathrm{mg}$ or greater per $100 \mathrm{ml}$ had higher rates of non-fatal myocardial infarctions and coronary deaths at all age ranges than did those with cholesterol levels less than $255 \mathrm{mg} / 100 \mathrm{ml}$. He also showed that subjects with mild hypertension had more coronary and cardiovascular episodes than did subjects with higher levels of blood pressure.

Though no statistically significant association was shown when each was assessed separately effort pain, a high cardiothoracic ratio, and E.C.G. abnormalities all occurred more often in the index group than in the controls. It is of interest that high prevalence rates for all these factors were shown in a large-scale community study of cardiovascular disorders in Jamaica (Fodor et al., 1964). Other studes in Jamaica have also shown an association of hypertension with certain personal factors, including parity, build, and heredity (Miall et al., 1962), all of which may therefore be assumed to be likely risk factors.

All the evidence, therefore, seems to justify strenuous efforts to identify subjects with only mildly raised blood pressure who may be at high risk. In order to arrive at adequate criteria for this identification a long-term follow-up of large numbers of subjects would be necessary. What is clear is that subjects with the same blood pressure levels do not have the same degree of risk. Heredity, diet, social habits, and a wide range of factors must play a part. Dollery (1973) pointed out that the therapy of hypertension has achieved the "first substantial success in the control of a common non-infectious disease." Clearly, the problem of discovering the full number of those who would benefit from treatment deserves much thought.

The need for further information on mild hypertension in West Indian patients has been emphasized by studies which show $(a)$ the high levels of all degrees of hypertension in defined communities in Jamaica (Miall et al., 1962) and in St. Kitts (Schneckloth et al., 1962); (b) the rapidity with which many Jamaican patients with initially mild hypertension show a rise to known high-risk levels, and the relative ease with which early treatment may prevent this rise (Stuart et al., 1972); and (c) the unexpectedly high incidence of E.C.G. and radiological evidence of left ventricular hypertrophy in subjects with only mild increases of blood pressure (Stuart et al.,
1972). The main problem is the establishment of criteria for the recognition of higher-risk subjects (see chart).

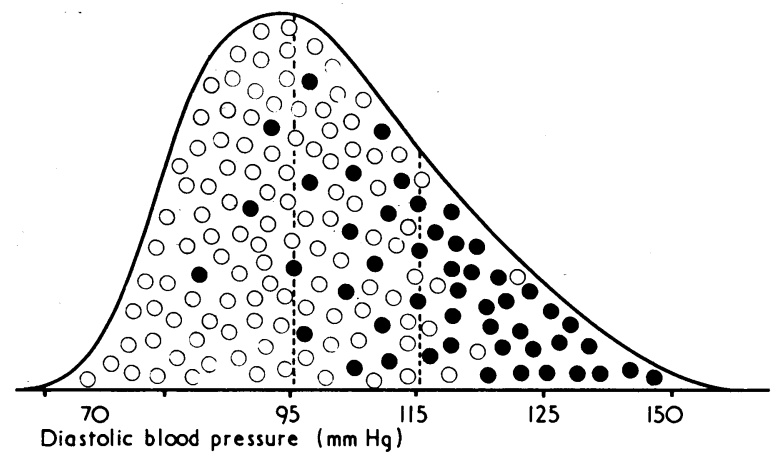

Schematic representation of problem of mild hypertension. Black circles indicate persons with moderate hypertension or those in whom, hypothetically, morbid or fatal events due to hypertension will eventually occur. Open circles indicate rest of the population. The question is to find data that could be used, in addition to diastolic blood pressure, to single out the black circles. Adapted from Strasser (1972).

Our data suggest that the presence of two or more of the risk factors examined might have been of some value in assessing prognosis in our subjects. There must be many others. The following general inferences appear to be justified: (a) that it should be practicable to set up criteria for establishing "risk" profiles based on the presence at the initial examination of a number of factors already considered or shown by future studies to be associated with an adverse prognosis, and (b) that persons with low-risk scores could be kept under observation, allowing treatment to be concentrated on the relatively small numbers of subjects in the high-risk categories. This would not only rationalize therapy but would reduce the dimension of such treatment programmes to more practicable levels. Indeed, it is probable that it is the impracticable nature of the problem that has prevented programmes of this nature from being undertaken in the past. It would also reduce the inescapable discomfort and inconvenience caused to patients to more acceptable and justifiable levels.

Our numbers were too small to establish high levels of sensitivity or specificity for the predictors studied (table III). It is, however, clearly indicated that this might be achieved by future long-term study of these and other probable predictors in larger numbers of subjects.

Though our analysis was not designed to test physical activity, smoking habits, heredity, emotional or psychological characteristics, and a number of other possible risk factors it outlines a practical approach to one of the most important public health problems of our time. Final answers must clearly await the results of studies of much larger numbers carried out over longer periods. In medicine, however, as in many of the other practical affairs of life it is often desirable to act on commonsense deductions from reasonable premises while awaiting final proof of a hypothesis which may require timeconsuming study.

It is a pleasure to record our thanks to Dr. W. E. Miall and Dr. A. Davis, the past and present heads of the Medical Research Council's Epidemiological Research Unit, for permission to make use of the data from their surveys in Lawrence Tavern.

\section{References}

Dollery, C. T. (1973). British Medical Bulletin, 29, 158.

Evans, J. G., and Rose, G. (1971). British Medical Bulletin, 27, 37. 
Fodor, J., Mial, W. E., Standard, K. L., Fejfar, Z., and Stuart, K. L. (1964). Bulletin of the World Health Organization, 31, 321.

MacIntyre, I., and Ralston, M. (1954). Biochemical fournal, 56, xliii.

Miall, W. E., Kass, E. H., Ling, J., and Stuart, K. L. (1962). British Medical fournal, 2, 1283.

Miall, W. E., et al., (1972). Bulletin of the World Health Organization, 46, 685. Paul, O. (1971). British Heart fournal, 33, Suppl., p. 116.

Pose, G. A. (1962). Bulletin of the World Health Organization, 27, 645.

Schneckloth, R. A., Stuart, K. L., Corcoran, A. C., and Moore, F. E. (1962). American Heart fournal, 63, 607.

Smirk, F. H. (1957). High Arterial Pressure. Oxford, Blackwell Scientific.
Society of Actuaries (1959). Build and Blood Pressure Study, vol. 1. Chicago, IIl., Society of Actuaries.

Sokolow, M., and Lyon, T. P. (1949). American Heart fournal, 37, 161.

Strasser, T. (1972). World Health Organization Meeting on Community Control of Stroke and Hypertension CVD/S/72.29.

Stuart, K. L., MacIver, C., and Nicholson, J. A. (1972). British Medical Fournal, 2, 21.

Veterans Administration Cooperative Study Group on Anti-hypertensive Agents (1967). Fournal of the American Medical Association, 202, 1028.

Veterans Administration Cooperative Study Group on Anti-hypertensive Agents (1970). Journal of the American Medical Association, 213, 1143.

\title{
Cryosurgery for Benign Cervical Lesions
}

\author{
J. E. PECK
}

British Medical fournal, 1974, 2, 198-199

\begin{abstract}
Summary
Fifty patients with cervical erosions causing symptoms were treated with cryosurgery. Healing was complete in $41(91 \%)$ out of 45 patients who were seen six weeks later, and in 21 patients who were followed up in greater detail the rate of symptomatic relief was high. All the patients who were treated without anaesthesia found the procedure acceptable. Only one patient noted postoperative bleeding, and this was minimal.
\end{abstract}

\section{Introduction}

Benign cervical erosions are usually treated by electrical or thermal diathermy. Such cauterization without sedation or analgesia is more often painful than many of those who use it care to admit, and many treatments are sometimes needed to secure complete healing. Ostergard et al. (1969) claimed that cryosurgery brought quicker healing of the cervix and gave a greater rate of cure (Ostergard et al., 1969; Jackson, 1972). Miller and Elstein (1973) were unable to show these advantages, but they limited application of the cryoprobe to a two-minute period. This paper reports the results of cryosurgery in $\mathbf{5 0}$ cases of benign erosion of the cervix.

\section{Patients and Methods}

All 50 patients had benign cervical lesions causing symptoms. Unsuspected malignant or premalignant conditions were excluded by cytological examination. Treatment was given at any time in the month except during menstruation. The cryoprobe used was the Spembley TCCIO with nitrous oxide as the coolant. The advantage of this gas is that it is readily available and cheap.

For outpatient treatment the cervix was exposed with a Cusco's speculum which also splints the cervix so that vulsellum forceps need not be used. The cryoprobe was applied to the cervix until a white rim of frozen tissue appeared and the freezing was then continued for a further one to three minutes. All patients were asked to attend for follow-up after six weeks and $\mathbf{4 5}$ of them did so. These were asked to return again if they had any further problems. The 21 patients treated most re-

Department of Obstetrics, Institute of Obstetrics and Gynaecology, Hammersmith Hospital, London W12 OHS

J. E. PECK, M.B., M.R.C.o.G., Registrar and Tutor (Present appointment: Lecturer in Obstetrics and Gynaecology, University of Leeds, St. James's Hospital, Leeds LS9 7TF) cently were assessed in greater detail, and only one of these defaulted.

\section{Results}

Healing.-Healing was complete in $41(91 \%)$ of the 45 patients seen six weeks after the operation. Of the 21 patients treated most recently (one defaulted) the cervix had healed completely in 19. The one patient with a poor result was completely cured after a second application of the cryoprobe. The time in the menstrual cycle when treatment was undertaken did not affect the cure rate.

Relief of Symptoms. - The 21 most recent patients were carefully assessed for symptomatic relief. Nine patients who had had intermenstrual or postcoital bleeding were all cured. Out of fifteen patients who had complained of vaginal discharge 13 were either cured or improved. Three patients out of four who complained of pelvic pain were cured, and the one patient with continuing pain had pre-existing pelvic inflammatory disease.

Pain.-Seven patients out of 21 had a general anaesthetic because of other surgery undertaken at the same time. Of the remaining 14 patients five noted slight discomfort in the lower abdomen during treatment. In no case was this severe and all five patients mentioned the discomfort only on direct questioning. One patient had pain after the operation, which was probably accounted for by pre-existing pelvic inflammatory disease.

Postoperative Discharge.-Seventeen out of 20 patients questioned observed a watery discharge for up to two weeks after treatment, but none found this distressing. All had been warned that they might have a transient discharge.

Haemorrhage.-Only one patient noted spotting postoperatively and there were no cases of frank secondary bleeding.

\section{Discussion}

Cryosurgery seems to be an advance in the outpatient treatment of benign cervical lesions. Ostergard et al. (1969), comparing its efficacy with that of diathermy, found a healing rate of $90 \%$ with cryosurgery and 33\% with diathermy. Jackson (1972) found a cure rate of $83 \%$ with cryosurgery compared with $62 \%$ with diathermy, but most of the patients were treated under general anaesthesia. Similar high cure rates with cryosurgery have been reported by others (Ostergard et al., 1968; Townsend et al., 1971; Young et al., 1972).

In the present series the healing rate improved with the increased experience of the operator. This seemed to be due to the use of longer freezing times in selected cases. As a standard treatment it was found best to freeze for two minutes after a white rim had appeared on the cervix all round the cryoprobe. With larger lesions the freezing time can be increased to three 